\title{
Stages en formation professionnelle du secondaire : perceptions des élèves de la qualité de la formation offerte en entreprise
}

Auteurs

Annie Dubeau, Canada,

dubeau.annie@uqam.ca

Mylène Beaulieu, Canada,

beaulieu.mylene.3@courrier.uqam.ca

Yves Chochard, Canada,

chochard.yves@uqam.ca

Isabelle Plante, Canada,

plante.isabelle@uqam.ca 


\section{REVUE HYBRIDE DE L'ÉDUCATION}

\section{Résumé}

Cet article présente les résultats d'une étude menée auprès de 192 élèves inscrits dans un programme de formation professionnelle au Québec visant à documenter leurs perceptions de la qualité de la formation reçue pendant un stage en entreprise. La qualité de la formation est décrite par quatre indicateurs - la planification du stage, le soutien apporté au stagiaire pendant le stage, la complexité et la variété des activités à réaliser, la rétroaction - qui permettent de conclure à la qualité et déterminer la manière dont la formation devrait être structurée. Comme la qualité de la formation est susceptible d'être modulée par la taille de l'entreprise dans laquelle le stagiaire réalise son stage, nous avons vérifié si les perceptions des stagiaires de la qualité de la formation variaient selon la taille de l'entreprise d'accueil.

Même si la planification des objectifs, des activités et des modalités du stage entourant le choix des tâches confiées au stagiaire est fortement susceptible d'améliorer la qualité de la formation en entreprise offerte pendant les stages, les résultats des analyses descriptives et de variance suggèrent que les élèves ne perçoivent pas nécessairement que les activités de formation sont planifiées à l'avance par le formateur de l'entreprise, et ce, de façon plus importante dans la moyenne et la petite entreprise. Ceci met en lumière différents aspects à considérer pour améliorer la qualité de la formation offerte en entreprise durant le stage et éventuellement prévenir l'abandon des stages et de la formation professionnelle par l'élève.

Mots-clés : qualité de la formation en entreprise ; formateur en entreprise ; stages ; alternance travail-études ; formation professionnelle du secondaire 


\section{$\&$}

\section{REVUE HYBRIDE DE L'ÉDUCATION}

\section{Problématique}

Dans la volonté d'offrir aux élèves des formations signifiantes et ancrées dans la pratique professionnelle, la plupart des programmes de formation professionnelle du secondaire du Québec proposent au moins un stage à réaliser en cours de formation. Dans la formule d'alternance travail-études (ATE), les stages en entreprise représentent au moins $20 \%$ de la durée du programme de formation (Lebel, 2002). Or, en dépit de la place accordée aux stages, plus d'un jeune sur cinq abandonne sa formation avant l'obtention du diplôme (OCDE, 2015). Qui plus est, des études de cas suggèrent que le choix d'abandonner la formation professionnelle apparaît typiquement pendant la période de stage, lorsqu'un problème de qualité de la formation apparaît (Lebel, 2002). Ce constat est pour le moins étonnant compte tenu des bénéfices associés à la mise en pratique des acquis sous la forme de stage (Veillette, 2004 ; Doray et Bastien, 2015).

Plusieurs facteurs liés au stagiaire ou à l'entreprise formatrice sont susceptibles d'affecter la qualité de la formation diffusée en milieu de travail et, par conséquent, de réduire la persévérance des personnes formées. Du côté du stagiaire, on sait qu'un manque de motivation, le stress lié au travail ou l'incapacité d'atteindre les objectifs d'apprentissage peuvent expliquer son choix d'abandonner le programme (Forsblom, Negrini, Gurtner et Schumann, 2016 ; Stalder et Schmid, 2006).

Du côté de l'entreprise, la faible qualité de la formation offerte en milieu de travail peut entraîner la rupture de l'entente de stage avec l'entreprise formatrice (Negrini, Forsblom, Gurtner et Schumann, 2016). Un problème de qualité peut apparaître avant que la formation ne débute, à l'étape du recrutement du stagiaire par l'entreprise. Par exemple, en Suisse, plusieurs organisations s'appuient sur les résultats d'un test composé de questions à choix multiples (le multicheck) pour choisir leurs stagiaires, alors que, selon Siegenthaler (2011), ce test ne prédit pas la réussite de la formation. En Australie, Dickie, McDonald et Pedic (2011) ont observé que les entreprises qui présentaient les plus hauts taux d'abandon des stagiaires sont celles qui n'avaient pas établi de lignes directrices pour leur processus de sélection des stagiaires. En outre, d'après Forsblom, Negrini, Gurtner et Schumann (2016), près de $80 \%$ des entreprises ne perçoivent pas ce problème et sont convaincues de suivre un processus de recrutement approprié pour assurer la formation de stagiaires.

De plus, il appert que la persévérance dans les stages serait aussi influencée par la taille de l'entreprise d'accueil, elle-même déterminée par le nombre de personnes qui y travaillent à plein temps. Selon l'étude allemande de Rohrbach-Schmidt et Uhly (2015), la probabilité que les personnes formées aillent jusqu'au bout de leur stage est de 18,3\% plus élevée dans les entreprises de plus de 300 salariés que dans les 


\section{6}

\section{REVUE HYBRIDE DE L'ÉDUCATION}

entreprises de sept salariés et moins. Cette différence s'explique par le fait que les grandes entreprises sont dotées d'une unité de ressources humaines et d'une infrastructure de formation, ce qui permet généralement à ces entreprises d'offrir une formation de meilleure qualité. Au Québec, près de $98 \%$ des entreprises emploient moins de 100 personnes (Institut de la statistique du Québec, 2014) et il semble que, dans la Belle Province, la taille de l'entreprise joue également un rôle sur la diplomation, comme le démontrent les analyses multivariées de Gaudreault, Morin, Simard, Perron et Veillette (2018). Ces chercheurs ont mis en évidence que la taille des entreprises était un facteur significativement associé aux taux de diplomation et de qualification d'élèves ayant accompli au moins sept ans d'école secondaire. Ils ont constaté que les territoires caractérisés par une forte présence d'entreprises de moins de 10 employés ou de plus de 200 employés étaient associés à un taux de diplomation plus faible. Toutefois, leur étude ne permet pas de déterminer comment la taille de l'entreprise agit sur la diplomation et si ce phénomène s'observe aussi dans le contexte spécifique de la formation en alternance travail études.

En somme, on sait que la taille des entreprises influence la diplomation, mais on ignore si la qualité du stage joue un rôle dans cette relation. On ne sait pas non plus de quelle façon les stagiaires en formation professionnelle perçoivent la qualité de la formation en milieu de travail et si cette qualité augmente avec la taille de l'entreprise. Ceci est d'autant plus important compte tenu du fait qu'un dispositif de formation en entreprise mal structuré peut entraîner une fin prématurée du stage.

Cette question revêt un enjeu social important, car la formule de l'alternance travail-études est actuellement perçue comme une réponse adaptée au phénomène de pénurie de main-d'œuvre qui touche plusieurs secteurs économiques du Québec (Ampuy, 2019; Bourdua, 2019; Lévesque, 2019; Rainville, 2019). Plusieurs chercheurs québécois partagent ce constat et considèrent que, lorsque le dispositif d'alternance s'appuie sur un accompagnement en entreprise de qualité, il est en mesure de répondre à la fois aux besoins de main-d'œuvre des entreprises et aux enjeux de réussite scolaire des stagiaires (Gagnon, 2007; Hardy et Ménard, 2008 ; Mazalon et al., 2010 ; Roy, 2015).

\section{Cadre théorique}

La qualité de la formation en entreprise est souvent évaluée au moyen de modèles fondés sur l'adaptation à l'objectif visé (c.-à-d., fit-forpurpose approach, voir Harvey et Green, 1993) qui sont principalement centrés sur la promotion de l'apprentissage et le développement des compétences des élèves (Negrini et al., 2016). Selon ces modèles, la qualité d'un dispositif de formation repose sur la capacité d'un intervenant à intégrer le point de vue des apprenants dans ses pratiques pédagogiques. En contexte scolaire, la pertinence de tenir compte du point de vue de l'élève par le biais de ses perceptions a été largement démontrée 


\section{$\&$}

\section{REVUE HYBRIDE DE L'ÉDUCATION}

(Gregory, Allen, Mikami, Hafen et Pianta, 2014 ; Ross, 1992 ; Schunk et Mecee, 1992 ;Sinclair et Fraser, 2002). Par exemple, Ricard et al. (2014) ont remarqué que les élèves d'une même classe s'entendaient entre eux sur les pratiques de leur enseignant et que leurs perceptions coïncidaient avec l'autoévaluation de cet enseignant. En outre, de nombreux modèles théoriques, pour comprendre l'apprentissage et l'expérience scolaire (voir Bandura, 1986 ; Eccles et Wigfield, 2002), sont construits sur le postulat que ce n'est pas la réalité qui détermine les comportements de l'élève, mais bien ses perceptions et son interprétation des situations auxquelles il fait face (Neuville, 2006).

Pour examiner la qualité de la formation en entreprise, plusieurs études empiriques ciblent les quatre dimensions qui composent le modèle développé par Negrini et al. (2016) (figure 1). Nous décrivons maintenant ces dimensions.

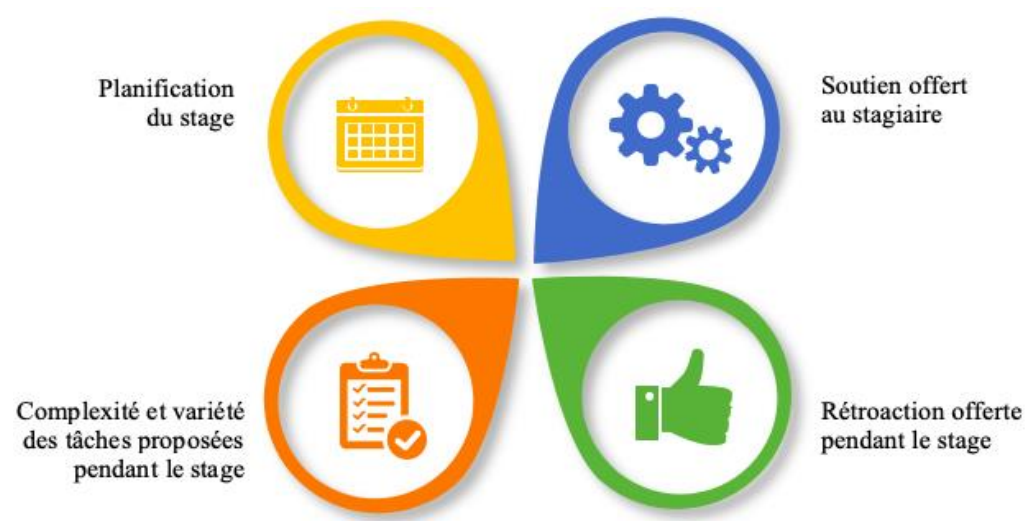

Figure 1 : Modèle de la qualité de la formation en entreprise pendant le stage, adapté de Negrini et al., 2016.

\section{Perceptions des élèves de la planification du stage}

La première dimension du modèle, la planification des activités de formation, réfère au degré de préparation du superviseur et aux paramètres que ce dernier intègre dans sa planification de la formation en entreprise (Marzano et al., 2000). Une préparation soignée devrait faire appel à la contribution de l'élève. Pour ce faire, le superviseur devrait convenir avec lui des objectifs qui seront poursuivis pendant le stage et prévoir à l'avance des activités d'apprentissage en lien avec les objectifs à atteindre. II devrait prendre connaissance des attentes du stage (niveau de compétence à atteindre au terme du stage) puis informer le stagiaire des étapes et des apprentissages à réaliser (Negrini et al., 2016). 


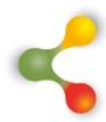

\section{REVUE HYBRIDE DE L'ÉDUCATION}

\section{Perceptions de l'élève du soutien offert pendant le stage}

La deuxième caractéristique de la qualité de la formation en entreprise correspond aux perceptions de l'élève du soutien offert pendant le stage. Le soutien offert par le superviseur et les autres personnes de l'entreprise devrait être orienté vers les apprentissages et le développement de compétences. Pour ce faire, le superviseur recourt à diverses stratégies comme l'explicitation des raisons qui sous-tendent la réalisation d'une tâche ou l'établissement de liens entre les explications fournies. II s'assure également que le stagiaire puisse réaliser plus d'une fois les nouvelles tâches qui lui sont confiées, il lui laisse suffisamment de temps de pratique et l'amène à trouver ses propres solutions pour réaliser les tâches qui lui sont confiées.

\section{Perceptions de l'élève de la complexité et de la variété des tâches proposées pendant le stage}

La troisième dimension du modèle de la qualité de la formation en entreprise est la complexité et la variété des tâches proposées pendant le stage. Cette dimension se réfère aux tâches dans lesquelles le stagiaire met à profit ses connaissances et démontre ses compétences. Pour ce faire, le superviseur confie au stagiaire des tâches variées qu'il peut réaliser seul. II lui offre également des opportunités d'apprendre. II lui propose des tâches où le stagiaire peut travailler de manière autonome et où il peut prendre seul certaines décisions (Negrini et al., 2016). Ces tâches devraient être importantes pour l'entreprise et suffisamment complexes pour susciter un apprentissage.

\section{Perceptions des élèves de la rétroaction offerte pendant le stage}

La quatrième dimension de qualité réfère aux perceptions du stagiaire de la rétroaction offerte pendant le stage. C'est en offrant une rétroaction fréquente, spécifique et appropriée sur la valeur et la qualité du travail que le superviseur contribue à la préservation et au renforcement de l'estime de soi du stagiaire (Rivard, 2000). Au contraire, une rétroaction insuffisante peut produire un affaiblissement de la motivation et réduire l'engagement du stagiaire envers l'entreprise d'accueil (Eraut, 2004). La rétroaction peut prendre la forme de renforcements positifs, de commentaires ou d'entretiens réguliers avec le superviseur.

En somme, selon la structure proposée par Negrini et al. (2016), un superviseur offre une formation de qualité pendant le stage de son étudiant (1) en effectuant une bonne planification du stage, (2) en offrant du soutien au stagiaire, (3) en proposant des activités d'apprentissage complexes et variées, et (4) en produisant une rétroaction régulière sur le travail du stagiaire. 


\section{8}

\section{REVUE HYBRIDE DE L'ÉDUCATION}

\section{La présente étude}

Le but de la présente étude est d'adapter les quatre dimensions du modèle de Negrini et al. (2016) afin de documenter la qualité perçue des stages en entreprise en contexte d'alternance travail-études et selon le point de vue d'élèves québécois. Elle examine aussi si les perceptions de ces élèves varient selon la taille de l'entreprise d'accueil.

\section{Méthodologie}

\section{Participants et procédure}

L'échantillon est composé de 192 élèves qui proviennent de treize centres de formation professionnelle (FP) répartis dans dix commissions scolaires (CS) du Québec : la CS de l'Or et des Bois, la CS de la Capitale, la CS de Laval, la CS des Affluents, la CS des Rives-du-Saguenay, la CS du Val-des-Cerfs, la CS de Jonquière, la CS du Lac-Saint-Jean, la CS Marie-Victorin et la CS de la Seigneurie-des-Milles-Îles. Les élèves sont issus de plus de dix programmes de formation professionnelle de courte durée tels que les diplômes d'études professionnelles en vente-conseil ( $40 \%$ des élèves), en vente de voyage (18\%), en boulangerie (10\%), en boucherie $(8 \%)$, en comptabilité $(8 \%)$, en conduite de machines de traitement du minerai $(4 \%)$, en entretien général d'immeuble $(3 \%)$, en conseil technique en entretien (3\%), en horticulture et jardinerie $(3 \%)$, et l'attestation de spécialisation professionnelle en représentation (3\%).

En conformité avec les exigences éthiques établies par le Comité institutionnel d'éthique de la recherche avec des êtres humains (CIEREH), seuls les élèves qui ont donné leur consentement par écrit pour prendre part à l'étude ont participé à la recherche. La passation du questionnaire s'est déroulée en salle de classe au retour du premier stage en milieu de travail. Le temps de complétion des questionnaires n'a pas excédé 20 minutes.

\section{Mesures}

Pour documenter les perceptions des élèves de la qualité de la formation offerte en entreprise pendant un stage, un instrument de mesure basé sur les quatre dimensions de la qualité de la formation en entreprise décrites dans le cadre théorique a été utilisé. Puisqu'aucun outil comparable n'était disponible en langue française, cet instrument de mesure a été sélectionné en raison de ses bonnes qualités psychométriques.

Initialement développés et validés en langue allemande, les items de cet instrument de mesure ont été traduits en français et adaptés au contexte de formation québécois afin de mesurer la qualité de la formation 


\section{$\&$}

\section{REVUE HYBRIDE DE L'ÉDUCATION}

en entreprise auprès de stagiaires de la formation professionnelle du secondaire.

La mesure de la planification des activités de formation par le superviseur de stage comprend 3 items $(\alpha=0,87)$ et évalue la perception du stagiaire envers la préparation et la planification de la formation du superviseur de stage. Le soutien offert durant le stage a été mesuré à l'aide d'une échelle de 7 items $(\alpha=0,83)$ évaluant le soutien offert par le réseautage dans l'entreprise et l'autorégulation au travail du stagiaire, tandis que la dimension "complexité et la variété des tâches proposées pendant le stage " a été mesurée par une échelle de 9 items $(\alpha=0,92)$ pour rendre compte de l'activation cognitive chez les élèves en formation. Finalement, la rétroaction offerte pendant le stage a été mesurée par une échelle comportant 6 items $(\alpha=0,94)$ et permettant de déterminer la perception du stagiaire sur la rétroaction portant sur la valeur de son travail. Pour chacun des items, les participants étaient invités à indiquer jusqu'à quel point chaque énoncé correspondait à ce qu'ils ressentaient sur une échelle de type Likert de 1 (ne correspond pas du tout) à 7 (correspond parfaitement).

En complément, pour mesurer l'appréciation générale du stage, chaque élève devait rapporter son appréciation globale sur une échelle de Likert allant de 1 (niveau d'appréciation le plus faible) jusqu'à 10 (niveau d'appréciation le plus élevé).

Enfin, la taille de l'entreprise accueillant des stagiaires a été rapportée par les élèves dans le questionnaire. Ces derniers devaient estimer le nombre d'employés que pouvait compter leur milieu de stage.

\section{Résultats}

Les 192 élèves (43\% de femmes et $57 \%$ d'hommes) âgés de 15 à 62 ans (âge moyen : 23 ans ; écart-type : 8,18 ans ; médiane : 19 ans) ayant pris part à l'étude ont réalisé un stage dans une entreprise québécoise à l'automne 2017. II s'agissait du premier stage de leur programme de formation professionnelle du secondaire. La majorité des élèves de l'échantillon (31\%) a été accueillie dans une entreprise comptant moins de 10 employés. Plus de $49 \%$ de l'échantillon ont effectué leur stage dans une entreprise comptabilisant de 10 à 50 employés. La proportion des élèves ayant complété leur stage dans une entreprise avec plus de 50 employés s'élevait à $20 \%$.

\section{Perceptions des élèves de la qualité de la formation reçue en entreprise pendant leur stage}

Pour documenter les perceptions des élèves de la qualité de la formation, des analyses descriptives ont été réalisées pour les quatre dimensions (la planification, le soutien, la complexité et la variété des 


\section{$\&$}

\section{REVUE HYBRIDE DE L'ÉDUCATION}

activités ainsi que la rétroaction offerte au stagiaire). Les analyses descriptives présentées permettent, d'abord, d'examiner le score moyen accordé par les élèves dans chacune des dimensions. Puis, le résultat à chacun des items est également présenté afin de permettre de contextualiser les perceptions des élèves. Les résultats sont présentés dans le tableau 1.

La planification du stage. Concernant la fixation des objectifs du stage par le superviseur, les résultats des analyses descriptives ont révélé des scores moyens de 5,5 et 5,8 sur 7 . L'avis des élèves à propos de la planification des activités de formation par le superviseur s'élève quant à lui à 4,7 , un des scores les plus faibles observés. Ces résultats suggèrent que les superviseurs impliquent les stagiaires dans le choix des objectifs à atteindre pendant le stage, mais que les activités de formation ne sont pas toujours bien planifiées.

Le soutien offert pendant le stage. À propos du soutien qui leur est offert pendant leur stage, les élèves ont, en moyenne, évalué à 5,9 sur 7 les items qui concernaient (1) la démonstration par le superviseur des tâches à réaliser, (2) l'explication ou la justification des raisons qui soustendent l'emploi d'une procédure et (3) la pratique de supervision consistant à proposer au stagiaire des tâches, devoirs ou exercices qu'il est en mesure de réaliser seul.

Tableau 1 : Dimensions et items de l'Échelle de la qualité de la formation, alphas de Cronbach, score moyen et écart-type des dimensions

\begin{tabular}{|c|c|c|}
\hline Dimensions et items & $\alpha$ & $\begin{array}{l}\text { Score moyen } \\
\text { (écart-type) }\end{array}$ \\
\hline Planification du stage & 0,87 & $5,34(1,56)$ \\
\hline $\begin{array}{l}\text { Le superviseur de stage convient avec moi des } \\
\text { objectifs de mon stage. }\end{array}$ & & 5,82 \\
\hline $\begin{array}{l}\text { 2. Le superviseur de stage m'informe des objectifs de } \\
\text { mon stage. }\end{array}$ & & 5,51 \\
\hline $\begin{array}{l}\text { Le superviseur de stage planifie des activités de } \\
\text { formation à l'avance. }\end{array}$ & & 4,74 \\
\hline Soutien offert pendant le stage & 0,83 & $5,72(1,13)$ \\
\hline $\begin{array}{l}\text { 4. Le superviseur de stage établit clairement des liens } \\
\text { entre les sujets qu'il aborde. }\end{array}$ & & 5,56 \\
\hline $\begin{array}{l}\text { 5. En général, le superviseur de stage n'explique la } \\
\text { tâche qu'une seule fois. }\end{array}$ & & 4,36 \\
\hline
\end{tabular}




\section{REVUE HYBRIDE DE L'ÉDUCATION}

Lorsque le superviseur de stage fait la

6. démonstration d'une tâche, il explique pourquoi il la 5,93 réalise de cette façon.

7. Le superviseur de stage me confie des tâches, devoirs ou exercices que je peux réaliser seul(e). Le superviseur de stage me laisse trouver mes

8. propres solutions pour réaliser les tâches qu'il me confie.

Le superviseur de stage s'assure que je réalise

9. plusieurs fois une même tâche afin que je puisse ma pratiquer souvent.

Le superviseur de stage s'assure de me laisser de

10. temps pour pratiquer les nouvelles tâches qui me sont confiées.

Complexité et la variété des tâches proposées pendant le stage

11. Le superviseur de stage s'assure que les tâches qui me sont confiées sont variées.

Durant ce stage, j'ai la possibilité d'utiliser des compétences diversifiées.

beaucoup de tâches différentes.

14. Le superviseur de stage me confie des tâches qui correspondent à mes compétences.

15. Le superviseur de stage me confie des tâches qui m'amènent à me dépasser.

4,05

faibles pour leur offrir un soutien adapté.

Le superviseur de stage s'adapte au niveau des

17. stagiaires les plus performants en exigeant davantage d'eux.

Durant ce stage, on me confie des tâches avec des responsabilités.

19. Les tâches qu'on me confie sont importantes pour l'entreprise.

\section{Rétroaction offerte pendant le stage}

Je reçois régulièrement des commentaires sur les

20. résultats de mon travail de la part du superviseur de stage. 


\section{REVUE HYBRIDE DE L'ÉDUCATION}

Le superviseur de stage me donne régulièrement

21. des commentaires sur mes apprentissages et

progrès.

Le superviseur de stage s'entretient régulièrement

22. avec moi au sujet de mon travail.

Le superviseur de stage me montre de quelle façon

je peux m'améliorer.

Le superviseur de stage suit ma progression et

24. s'assure de l'atteinte des objectifs d'apprentissage

de mon stage.

25. Le superviseur de stage récompense les bons résultats.

Appréciation générale du stage

Échelle de 1 à 10 permettant aux élèves de rapporter leur appréciation générale de leur stage

Taille de l'entreprise d'accueil du stagiaire

Les élèves doivent estimer le nombre d'employés

dans leur milieu de stage

$\mathrm{N} / \mathrm{A}$

En moyenne, les stagiaires évaluent à 5,5 que, lorsque le superviseur de stage leur confie une tâche, il les laisse trouver leurs propres solutions. II s'assure également qu'ils puissent réaliser plusieurs fois une même tâche afin qu'ils puissent se pratiquer souvent et ils perçoivent que le superviseur de stage établit des liens clairs entre les sujets qu'il aborde. Ces deux items obtiennent un score de 5 . Les élèves jugent en moyenne à 5,8 que le superviseur de stage s'assure de leur laisser assez de temps pour essayer et pour pratiquer les nouvelles tâches qui leur sont confiées. En revanche, les élèves évaluent moins favorablement $(4,4)$ l'item proposant que le superviseur de stage explique la tâche plusieurs fois.

La complexité et la variété des tâches proposées pendant le stage. II s'agit de la dimension évaluée le plus faiblement par les élèves. Ces derniers estiment en moyenne à 5,8 que leur superviseur de stage leur confiait des tâches qui correspondaient à leurs compétences. La perception moyenne des élèves d'avoir la possibilité d'utiliser des compétences diversifiées, de se voir confier des tâches avec des responsabilités importantes pour l'entreprise durant leur stage est de 5,6. Les élèves ont perçu plus faiblement que les tâches qui leur étaient confiées pouvaient les amener à se dépasser $(5,3)$ ainsi que la possibilité de réaliser beaucoup de tâches différentes $(5,4)$. Il en va de même pour la variété des tâches qui leur étaient confiées durant le stage $(5,4)$. Les items de la dimension qui obtiennent les scores les plus faibles portent sur la capacité du superviseur 


\section{8}

\section{REVUE HYBRIDE DE L'ÉDUCATION}

à discriminer les stagiaires les plus performants des plus faibles (scores respectifs de 4,6 et 4,1$)$.

La rétroaction offerte pendant le stage. En ce qui a trait à la rétroaction offerte durant le stage, les élèves ont accordé un score de 5,5 sur trois items : l'item en lien avec la réception de commentaires sur les résultats du travail, celui sur le suivi de la progression et de l'atteinte des objectifs d'apprentissage ainsi que celui sur la capacité du superviseur à montrer à l'élève comment s'améliorer. En ce qui concerne la tenue d'entretiens réguliers au sujet de leur travail, les stagiaires ont évalué leur perception à 5,2 , tandis que l'obtention de commentaires en lien avec leurs apprentissages et leurs progrès durant le stage reçoit un score de 5,3.

Appréciation générale du stage. En moyenne, les élèves ont accordé un score de 7,95 sur 10 pour indiquer à quel point ils avaient apprécié leur stage.

Variation de la qualité perçue de la formation reçue pendant le stage en fonction de la taille de l'entreprise

En complément aux analyses descriptives menées, nous avons examiné si les perceptions des élèves de la qualité de la formation reçue en entreprise pendant le stage variaient selon la taille de l'entreprise. Pour ce faire, nous avons réalisé des analyses de variance (ANOVAs) afin de comparer les moyennes des groupes d'élèves établies sur la base de la taille de leur entreprise d'accueil. Trois groupes d'élèves ont été créés soit : (1) les élèves qui ont effectué un stage dans une entreprise de 10 employés et moins $(n=52),(2)$ ceux ayant fait un stage dans une entreprise de 10 à 50 employés $(n=83)$ et ceux ayant effectué un stage dans une entreprise de 50 employés et plus $(n=33)$. Les ANOVAs ont été réalisées en incluant une procédure de rééchantillonnage (Bootstrap, 1000 itérations ; intervalles de confiance à $95 \%$ ) afin de comparer les moyennes des groupes. Enfin, des analyses confirmatoires a posteriori de Bonferroni ont été menées afin de confirmer à quels endroits se situaient les différences. Dans ces analyses, nous avons introduit la satisfaction de l'élève dans son stage comme variable de contrôle afin d'éliminer son effet confondant sur la perception de la qualité de la formation en entreprise. Les résultats sont présentés dans le tableau 2. 


\section{REVUE HYBRIDE DE L'ÉDUCATION}

Tableau 2 : Moyenne (écart-type) des dimensions de la qualité de la formation perçue par les élèves, pour chaque groupe d'élèves établi sur la base de la taille d'entreprise et résultats des ANOVA pour les différences de moyenne entre les groupes

\begin{tabular}{|c|c|c|c|c|}
\hline & \multicolumn{2}{|c|}{$\begin{array}{l}\text { Taille de l'entreprise } \\
(n=171)\end{array}$} & \multirow[b]{2}{*}{$\begin{array}{l}\text { Grande } \\
\text { Plus de } \\
50 \\
\text { employé } \\
s \\
n=33\end{array}$} & \multirow[b]{2}{*}{ ANOVA } \\
\hline & $\begin{array}{l}\text { Petite } \\
\text { Moins de } \\
10 \\
\text { employés } \\
n=52\end{array}$ & $\begin{array}{l}\text { Moyenn } \\
\text { e } \\
10 \text { à } 50 \\
\text { employé } \\
\mathrm{s} \\
\mathrm{n}=83\end{array}$ & & \\
\hline \multicolumn{5}{|l|}{$\begin{array}{l}\text { Qualité de la } \\
\text { formation en } \\
\text { entreprise }\end{array}$} \\
\hline Planification & $\begin{array}{c}5,33 \\
(1,51) \mathrm{a}\end{array}$ & $\begin{array}{l}5,16 \\
(1,67)_{b}\end{array}$ & $\begin{array}{l}5,94 \\
(1,05) a, b\end{array}$ & $\mathrm{~F}(2,16)=5,00, \mathrm{p}=0,01$ \\
\hline Soutien & $5,80(1,13)$ & $\begin{array}{l}5,58 \\
(1,24)\end{array}$ & $\begin{array}{l}5,95 \\
(0,92)\end{array}$ & $F(2,164)=1,00, p=n s$ \\
\hline $\begin{array}{l}\text { Complexité et } \\
\text { variété } \\
\text { des tâches }\end{array}$ & $5,32(1,45)$ & $\begin{array}{l}5,05 \\
(1,41)\end{array}$ & $\begin{array}{l}5,63(1,34 \\
)\end{array}$ & $\mathrm{F}(2,164)=1,90, \mathrm{p}=n s$ \\
\hline Rétroaction & $5,49(1,50)$ & $\begin{array}{l}5,00 \\
(1,68)\end{array}$ & $\begin{array}{l}5,57 \\
(1,21)\end{array}$ & $\mathrm{F}(2,164)=1,63, \mathrm{p}=n s$ \\
\hline Qualité globale & $5,48(1,25)$ & $\begin{array}{l}5,20 \\
(1,35)_{a}\end{array}$ & $\begin{array}{l}5,77 \\
(1,01) a\end{array}$ & $F F(2,164)=3,14, p=0,05$ \\
\hline $\begin{array}{l}\text { Appréciation } \\
\text { globale du stage }\end{array}$ & $8,33(1,84)$ & $\begin{array}{l}7,75 \\
(2,60)\end{array}$ & $\begin{array}{l}8,09 \\
(1,83)\end{array}$ & $\mathrm{F}(2,165)=1,102, p=n s$ \\
\hline
\end{tabular}

Note : Les moyennes avec le même indice diffèrent significativement à $p<0,05$. La satisfaction de l'élève de son stage est incluse comme variable de contrôle dans l'analyse de la variance de la perception de la qualité de la formation en entreprise.

En somme, ce sont les élèves qui ont effectué un stage dans une entreprise de taille moyenne qui rapportent les perceptions de qualité les

Numéro $5 \quad 155 \quad$ Printemps 2021




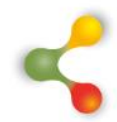

\section{REVUE HYBRIDE DE L'ÉDUCATION}

plus faibles, tant pour les quatre dimensions étudiées que pour l'appréciation globale du stage. Les perceptions des élèves entre les groupes d'entreprises diffèrent significativement pour la dimension de la planification de la formation et pour celle de la qualité globale perçue de la formation en entreprise. En effet, pour les autres dimensions de qualité, les analyses ne permettent pas de conclure à des différences statistiquement significatives en fonction de la taille de l'entreprise.

Spécifiquement, les analyses confirmatoires a posteriori de Bonferroni révèlent que la perception de la planification des activités de formation pendant le stage est statistiquement différente dans la grande entreprise comparativement à la petite et à la moyenne entreprise. C'est dans la grande entreprise que la planification du superviseur est perçue comme étant la plus organisée $(5,9)$. Quant à la qualité globale de la formation en entreprise, les résultats révèlent des différences statistiquement significatives entre les élèves qui ont réalisé un stage dans une grande et ceux qui l'ont réalisé dans une moyenne entreprise. Dans la moyenne entreprise, la qualité globale de la formation est plus faiblement perçue $(5,2)$.

\section{Discussion}

En synthèse, les résultats indiquent que les élèves ont l'impression que le superviseur prend le temps d'établir des objectifs liés au stage afin de planifier les activités de formation durant le stage. Toutefois, à propos de la planification des activités de formation, les élèves ne perçoivent pas nécessairement qu'elles sont planifiées à l'avance par le formateur de l'entreprise, et ce, de façon plus importante dans la moyenne entreprise. Ces résultats suggèrent que la planification des objectifs, des activités du stage et des modalités entourant le choix des tâches confiées au stagiaire est fortement susceptible d'améliorer la perception globale de la qualité de la formation en entreprise offerte pendant les stages.

Les élèves se sentent soutenus par le formateur en milieu de travail par le biais de ses actions et du degré de liberté que celui-ci leur accorde lors de l'accomplissement des tâches. Durant leur stage, les élèves jugent que le superviseur de stage a contribué à l'exploitation de leurs connaissances et au développement de leurs compétences. C'est en leur offrant des opportunités de réaliser des tâches variées et des tâches qui correspondent à leurs compétences que le superviseur y est arrivé. Ces perceptions sont similaires d'un milieu de stage à l'autre.

Une différence statiquement significative est notée chez les élèves ayant effectué un stage dans une grande entreprise. À l'instar des résultats de Rohrbach-Schmidt et Uhly (2015), il appert que les élèves de ce groupe se distinguent de ceux formés dans les petites et les moyennes entreprises. Des différences statistiques sont observées pour la dimension de la 


\section{$\&$}

\section{REVUE HYBRIDE DE L'ÉDUCATION}

planification du stage de même que pour la qualité globale de la formation offerte pendant le stage.

Indépendamment de la taille de l'entreprise d'accueil des stagiaires, les résultats révèlent également que, même si les élèves sont satisfaits de leur expérience de stage, ils reçoivent peu de rétroaction leur permettant d'améliorer leurs compétences pendant le stage. De ce fait, les élèves sont plus ou moins en accord avec le fait que le superviseur de stage s'entretient régulièrement avec eux au sujet du travail accompli. Le manque de renforcement positif à l'intention du stagiaire lors de l'obtention de bons résultats entraîne également le manque de rétroaction reçu par la stagiaire de la part du formateur en milieu de travail.

II importe de souligner que les analyses descriptives rapportées dans cet article ne nous permettent pas de capter l'importance relative des dimensions qui composent la qualité de la formation en entreprise de même que la relation de ces dimensions avec la persévérance et la réussite des élèves dans leur formation professionnelle. Cette limite pourrait être contournée par la réalisation d'une étude longitudinale. En effet, le suivi longitudinal d'élèves tout au long de leur formation permettrait d'évaluer plus précisément la contribution relative de la perception de la qualité de la formation dans le choix de l'élève de poursuivre ou d'abandonner sa formation professionnelle ; il s'agit là d'une piste de recherche prometteuse.

En terminant, rappelons que les résultats obtenus dans la présente étude ciblent des aspects à considérer pour bonifier la formation offerte aux formateurs en entreprise afin d'améliorer l'encadrement offert aux stagiaires de la formation professionnelle du secondaire pendant leur stage. II semble que des formations adaptées au contexte de l'entreprise d'accueil devraient être mises en place. En effet, il apparaît que les formateurs des entreprises sont confrontés à des situations spécifiques qui sont modulées par le type d'entreprise dans lequel ils œuvrent. À cet égard, les formateurs des entreprises de taille moyenne font face à des défis singuliers. Ce faisant, une offre de formation adaptée à leur réalité leur permettrait probablement de mettre en place des dispositifs de formation en entreprise de meilleure qualité. Ces aspects sont importants à considérer puisque les formateurs en entreprise sont des acteurs de première ligne impliqués dans la formation des élèves de la formation professionnelle du secondaire dont certains sont en quête d'un premier diplôme qualifiant. L'amélioration de la qualité des stages offerts à ces élèves pourrait permettre de prévenir leur abandon du stage et de leur formation professionnelle. La qualité de la formation en entreprise est donc une avenue incontournable à ne pas négliger afin de favoriser la persévérance et la réussite scolaires du plus grand nombre d'élèves engagés dans une formation professionnelle. 


\section{REVUE HYBRIDE DE L'ÉDUCATION}

\section{Références}

Ampuy, E. (2019). Le gouvernement mise sur les étudiants internationaux pour réduire la pénurie de main-d'œuvre. Quartier Libre. https://quartierlibre.ca/le-gouvernement-mise-sur-les-etudiantsinternationaux-pour-reduire-la-penurie-de-main-doeuvre/

Bandura, A. (1986). Social foundations of thought and action : A social cognitive theory. Prentice-Hall.

Bourdua, C. (2019). Pénurie de main-d'œuvre, mais où exactement?. Radio-Canada. https://ici.radio-canada.ca/nouvelle/1328050/penuriemain-oeuvre-apprentis-metiers-nord-ontario

Dickie, M., McDonald, R., et Pedic, F. (2011). A fair deal: apprentices and their employers in NSW: integrated research report. Sydney: NSW Board of Vocational Education and Training, Sydney.

Doray, P. et Bastien, N. (2015). Parcours scolaire en formation technique et alternance travail-études. Dans G. Boudesseul et al. (dir.), Alternance et professionnalisation, des atouts pour les parcours des jeunes et les carrières? (p. 139-152). Céreq.

Eccles, J.S. et Wigfield, A. (2002). Motivational beliefs, values, and goals. Annual Review of Psychology, 53(1), 109-132.

Eraut, M. (2004). Informal learning in the workplace. Studies in continuing education, 26(2), 247-273.

Forsblom, L., Negrini, L., Gurtner, J.L. et Schumann, S. (2016). Dropouts in Swiss vocational education and the effect of training companies' trainee selection methods. Journal of Vocational Education \& Training, 68(4), 399-415.

Gagnon, C. (2007). Arrimage des pratiques éducatives d'enseignants et de formateurs en entreprises en contexte d'alternance. Études de cas en formation professionnelle agricole. Recherches qualitatives, 27(1), 141-190.

Gaudreault, M., Morin, I., Simard, J. G., Perron, M., et Veillette, S. (2018). Les facteurs territoriaux de persévérance et de réussite scolaires au Québec. Les Sciences de l'éducation-Pour l'ère nouvelle, 51(3), 3760.

Gregory, A., Allen, J.P., Mikami, A.Y., Hafen, C.A. et Pianta, R.C. (2014). Effects of a professional development program on behavioral 


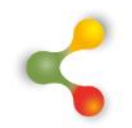

\section{REVUE HYBRIDE DE L’ÉDUCATION}

engagement of students in middle and high school. Psychology in the Schools, 51(2), 143-163.

Hardy, M., et Ménard, L. (2008). Alternance travail-études : les effets des stages dans la formation professionnelle des élèves. Revue des sciences de l'éducation, 34(3), 689-709.

Harvey, L. et Green, D. (1993). Defining quality. Assessment and Evaluation in Higher Education, 18(1), 9-26.

Institut de la statistique du Québec (2014). Science, technologie et innovation : Le financement et la croissance des petites et moyennes entreprises au Québec en 2014. Gouvernement du Québec. http://www.stat.gouv.qc.ca/statistiques/science-technologieinnovation/financement pme/financement-pme-2014.pdf

Lebel, D. (2002, octobre). Rapport d'évaluation du Programme de soutien financier à l'alternance travail-études, de 1998-1999 à 2000-2001. Ministère de l'éducation du Québec.

Lévesque, N. (2019, 18 août). Pénurie de main-d'œuvre... des solutions existent. le Soleil. https://www.lesoleil.com/opinions/point-devue/penurie-de-main-doeuvre-des-solutions-existentb3859aaf1617f973472f1ad39284c15d

Marzano, R.J., Gaddy, B.B., et Dean, C. (2000). What Works in Classroom Instruction. McReL.

Mazalon, É., Beaucher, C., et Langlois, M. (2010). L'évolution et la détermination des projets d'élèves inscrits dans un programme de formation professionnelle en alternance travail-études. Revue de recherche appliquée sur l'apprentissage, 3(10), 1-20.

Mazalon, É., Gagnon, C. et Roy, S. (2014). L'encadrement des stagiaires en milieu de travail: Étude exploratoire dans un cadre formel d'alternance en formation professionnelle initiale. Éducation et francophonie, 42(1), 113-135

Negrini, L., Forsblom, L., Gurtner, J.-L. et Schumann, S. (2016). Is There a Relationship between Training Quality and Premature Contract Terminations in VET?. Vocations and Learning, 9(3), 361-378

Neuville, S. (2006). La valeur perçue des activités d'apprentissage : quels en sont les sources et les effets ?. Dans B. Galand et É. Bourgeois 


\section{REVUE HYBRIDE DE L'ÉDUCATION}

(dir.), (Se) Motiver à apprendre (p. 85-96). Presses universitaires de France.

OCDE. (2015). Perspectives de l'OCDE sur les compétences 2015. Les jeunes, les compétences et l'employabilité. OCDE.

Rainville, P. (2019). Pour contrer la pénurie de main-d'œuvre [VIDÉO]. Le Quotidien. $\quad$ https://www.lequotidien.com/affaires/pour-contrer-lapenurie-de-main-doeuvre-video296afb3bb5ea86dd7dbb4f752aae8fd9

Ricard, V., Dion, E., Doré, R., Fortier, M.P., Dupéré, V. et Cantin, S. (2014). Les actions de l'enseignant de classe ordinaire et l'intégration sociale de l'élève présentant une déficience intellectuelle. Enfance en difficulté, 3, 5-24.

Rivard, P. (2000) La gestion de la formation en entreprise : Pour préserver et accroitre le capital compétence de votre organisation. Les Presses de I'Université du Québec.

Rohrbach-Schmidt, D. et Uhly, A., (2015). Determinanten vorzeitiger Lösung von Ausbildungsverträgen und berufliche Segmentierung im dualen System. [Déterminants de la résiliation anticipée des contrats de formation et de la segmentation professionnelle dans le système dual. Eine Mehrebenenanalyse auf Basis der Berufsbildungsstatistik. [Une analyse à plusieurs niveaux basée sur les statistiques de la formation professionnelle] Kölner Zeitschrift für Soziologie und Sozialpsychologie, 67(1), 105-134.

Ross, J.A. (1992). Teacher efficacy and the effects of coaching on student achievement. Canadian Journal of Education/Revue canadienne de l'éducation, 17(1), 51-65. DOI : 10.2307/1495395

Roy, S. (2015). Accompagnement de Stagiaires Dans Le Milieu de Travail en Contexte D'alternance en Formation Professionnelle Au Québec [thèse de doctorat inédite], Université de Sherbrooke.

Schunk, D.H. et Meece, J.L. (2006). Self-efficacy development in adolescence. Self-efficacy beliefs of adolescents, 5, 71-96.

Siegenthaler, F. (2011), Visualizing Transformation: Contemporary Art in Johannesburg [thèse de doctorat inédite], Universität Basel. 


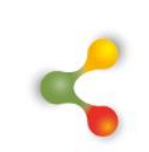

\section{REVUE HYBRIDE DE L'ÉDUCATION}

Sinclair, B.B. et Fraser, B.J. (2002). Changing classroom environments in urban middle schools. Learning Environments Research, 5(3), 301 328.

Stalder, B.E., Schmid, E., et Schmid, E. (2006). Lehrvertragsauflösungen, ihre Ursachen und Konsequenzen: Ergebnisse aus dem Projekt LEVA. [Les contrats d'apprentissage, leurs causes et leurs conséquences : résultats du projet LEVA]. Erziehungsdirektion des Kantons Bern, Bildungsplanung und Evaluation. [Direction de l'éducation du canton de Berne, Planification et évaluation de l'éducation].

Veillette, S. (2004). L'alternance travail-études au collégial : Les effets sur la réussite scolaire et l'insertion professionnelle [thèse de doctorat inédite], Université Laval. 